\section{Fortgeschrittenes Endometriumkarzinom: Chemotherapie wirkt besser als Bestrahlung}

\begin{abstract}
Hat der Krebs das zervikale Stroma erfasst, verdüstert sich die Prognose beim fortgeschrittenen Endometriumkarzinom. Mit Chemotherapie ist den meisten dieser Frauen besser geholfen als mit abdomineller Radiotherapie.
\end{abstract}

D ie Studie 122 der Gynecologic-Oncology-Group (GOG) hatte 2006 ergeben, dass fünf Jahre nach Chemotherapie (Doxorubicin/Cisplatin) noch 55\% der Patientinnen mit fortgeschrittenem Endometriumkarzinom (FIGO-Stadien III und IV) am Leben sind, von den Patientinnen, die eine Ganz-Abdomen-Bestrahlung erhalten hatten, nur $42 \%$ - ein signifikant schlechteres Ergebnis. Um herauszufinden, ob die Überlegenheit der Chemotherapie an bestimmte pathologische Merkmale der Tumorerkrankung geknüpft ist, haben die Autoren die GOG122-Daten noch einmal retrospektiv hinsichtlich des progressionsfreien und des Gesamtüberlebens analysiert.

In die Ergebnisse gingen die Angaben zu 396 Patientinnen ein: 202 hatten eine Bestrahlung, 194 eine Chemotherapie erhalten. War der Tumor ins zervikale Stroma eingebrochen, erhöhte dies das Progressions- bzw. Sterberisiko während der Nachbeobachtungszeit (im Median 74 Monate) um $44 \%$. Für den Befall der Lymphknoten ergab sich ein Trend hin zu einer 7\%igen Erhöhung des Progressions-/Sterberisikos pro positivem Knoten.

Für den lymphovaskulären Befall und den Tumoreinbruch ins zervikale Stroma errechnete sich sowohl für das progressionsfreie als auch für das Gesamtüberleben ein Trend zugunsten eines Nutzens der Chemotherapie mit Doxorubicin und Cisplatin. Die Größenordnung des Vorteils im Vergleich zur Bestrahlung lag zwischen 5 und $25 \%$. Doch die Konfidenzintervalle der Hazard Ratios schlossen den Wert 1 ein (Abb.). Es ist damit statistisch gesehen nicht ausreichend wahrscheinlich, dass die berechneten Werte einer tatsächlichen Differenz entsprechen.

Fazit: Die retrospektive Aufarbeitung der GOG-122-Studie unterstützt zwar die seit 2006 bekannten Ergebnisse. Sie liefert aber keine Belege für die Hypothese, wonach die Vorteile der Chemo- gegenüber der Strahlentherapie auf Faktoren der Tumorpathologie zurückgehen würden, als da wären: Befall des lymphovaskulären Raums, des zervikalen Stromas oder der pelvinen Lymphknoten. Robert Bublak

Tewari KS et al. Association of number of positive nodes and cervical stroma invasion with outcome of advanced endometrial cancer treated with chemotherapy or whole abdominal irradiation: a Gynecologic Oncology Group study. Gynecol Oncol. 2012;125(1): 87-93.

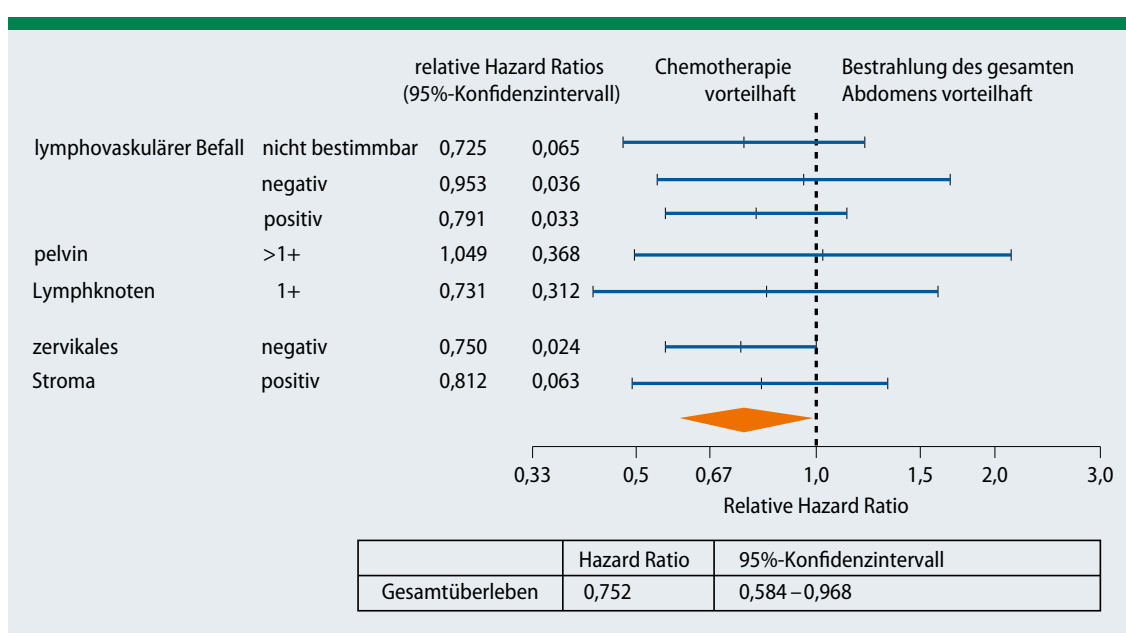

Abb.: Faktoren der Tumorpathologie beeinflussen möglicherweise den Therapieerfolg bei Patientinnen mit fortgeschrittenem Endometriumkarzinom
Kommentar von Prof. Mallmann: Die GOG122-Studie, die erstmalig 2006 die Überlegenheit einer adjuvanten Chemotherapie gegenüber einer Bestrahlung beim fortgeschrittenen Endometriumkarzinom dokumentierte, wies eine Reihe viel diskutierter Schwächen auf. So handelte es sich entgegen der primären Absicht nicht um eine reine Adjuvanzstudie, da $16 \%$ aller Frauen auch nach der Operation residuale Tumoren mit einer Größe von über $2 \mathrm{~cm}$ aufwiesen. Erhebliche Kritik kam auch bezüglich der Strahlentherapie, da im Rahmen dieser Studie eine in der adjuvanten Situation unübliche alleinige GanzAbdomen-Bestrahlung mit 30 Gy und einem Boost im kleinen Becken von 15 Gy erfolgte. Kritisiert wurde ferner die hohe Toxizität, die bei $17 \%$ der Patientinnen zu einem Studienabbruch führte. Mit der hier vorgestellten Re-Evaluation sollte überprüft werden, ob man mit den Parametern Lymph- oder Hämangiosis, Befall des Zervix-Stromas und Zahl der befallenen Lymphknoten ein Risikokollektiv definieren kann, das besonders von einer adjuvanten Chemotherapie profitiert.

„Insbesondere Patientinnen mit Lymphknotenmetastasen profitieren von einer adjuvanten Chemotherapie."

Die prognostische Bedeutung dieser Parameter konnte in dieser Re-Evaluation nochmals bestätigt werden und es wurde ergänzend gezeigt, dass Patientinnen mit zervikaler Stromainvasion in besonderem Maße von einer adjuvanten Chemotherapie profitieren.

Diese Ergebnisse sind mittlerweile durch die vorgelegte Meta-Analyse zur Wertigkeit der adjuvanten Chemotherapie beim Endometriumkarzinom überholt worden. In dieser aktuellen Meta-Analyse wird die in den Leitlinien bereits festgelegte Vorgehensweise nun nochmals bestätigt: Patientinnen mit fortgeschrittenem Endometriumkarzinom, insbesondere mit Lymphknotenmetastasen, profitieren von einer adjuvanten Chemotherapie mit Platin und Taxol. Die optimale Behandlung in dieser Situation besteht offenbar in der Kombination einer adjuvanten Chemotherapie mit einer Bestrahlung.

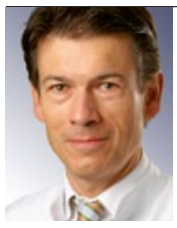

Prof. Dr. Peter Mallmann

Klinik für Frauenheilkunde und Geburtshilfe, Klinikum der Universität zu Köln peter.mallmann@uk-koeln. de 